\title{
Knowledge, Attitude and Practices Regarding COVID-19 among Postgraduate Trainees and House Officers
}

\section{Shandana Tarique, ${ }^{1}$ Shahid Sarwar ${ }^{2}$}

\begin{abstract}
Objective: To assess the knowledge, attitude and practice among house officers and post graduate trainees towards COVID-19 in tertiary care hospitals.

Methods: An online questionnaire was used as research tool to collect data from 115 house officers and post graduate trainees working in tertiary care hospitals. Questionnaire was divided into three sections. Knowledge was assessed on basis of choosing the best option, attitude was evaluated with agree/disagree and practices were considered on yes/no format. Data was analyzed with SPSS 24®(Armonk NY:IBM corp).

Results: 115 doctors responded to online survey. Mean age was 26.92 $\pm 2.92,62(53.9 \%)$ were female and $53(46.1 \%)$ were male. Regarding the status of participants, $46(40 \%)$ were house officers and $69(60 \%)$ were post graduate residents. Considering the domain of knowledge, maximum score was 12 and minimum was 4.Mean score was 8.75. It was observed that $82(71.3 \%)$ participants agreed that COVID-19 will be successfully controlled, while $114(99.1 \%)$ of participants counselled patients and their attendants about preventive measures. Regarding practice domain, 110(95.7\%) participants replied in affirmative that they wore mask when leaving home while 19(16.5\%) had undergone training for nasopharyngeal sample collection. Only 45(39.1\%) had undergone training for doffing and donning.

Pearson correlation was applied between the three domains and status of doctors. It was observed that postgraduate residents were significantly better in Covid-19 related knowledge $(\mathrm{p}<.05)$. Among the three domains, no significant relationship was observed.

Conclusion: Doctors have positive attitude though factors have been identified in knowledge and practice that need improvement.

Key words: COVID-19, knowledge, attitude, practice

How to cite: Tarique S., Sarwar S., Knowledge, Attitude and Practices regarding COVID-19 among Postgraduate Trainees and House Officers. Esculapio 2021;17(02):180-183.
\end{abstract}

DOI: https://doi.org/10.51273/esc21.2517214

\section{Introduction}

$\mathrm{E}$ mergence of infection due to novel corona virus has drastically modified life style globally. In December 2019, a new strain of virus was reported to be causing an outbreak in city of Wuhan in China. It was reported to cause pneumonia like illness with certain radiological and hematological characteristics. ${ }^{1}$ This disease is labelled in nomenclature as coronavirus

1. Shandana Tarique

2. Shahid Sarwar

1,2. Department of Medicine, Allama Iqbal Medical College, Lahore

\section{Correspondence:}

Dr.Shandana Tarique, Associate Professor of Medicine, Allama Iqbal Medical College, Lahore.E-mail: shandanatarique@gmail.com disease (COVID-19) by WHO and the reference name given to the virus is severe acute respiratory syndrome coronavirus 2 (SARS-CoV-2). ${ }^{2}$ Its rapid global spread led World Health Organization (WHO) to declare it as pandemic. Its high virulence and contagious nature made it major health emergency world-wide.

Corona virus are group of virus that cause diseases primarily related to respiratory symptoms. They are single stranded RNA virus. Novel virus is new version of SARS virus. It is considered that constant evolutionary process within different species or exposure to host environment, by mutation and eventual adaptation to a susceptible host has led to the new form of the viable pathogen. ${ }^{3}$

According to WHO, as of $23^{\text {rd }}$ February 2021; 223 
countries have been affected, 111279860 confirmed cases have been reported and death toll stands at 2466639 cases $^{4}$. Pakistan reported its first case on 26th February 2020. By $23^{\text {rd }}$ February 2021, Pakistan has more than five lakh confirmed cases. ${ }^{5}$

In event of major outbreaks, health care system has to bear the brunt. Health care workers form the backbone of a health facility. They are on the frontline and have to be prepared to deal with such emergencies. Doctors play critical role in management of these patients. Given the contagious nature of this disease, they themselves become vulnerable target. The exposure to the virus can lead to illness in doctors and unfortunately can prove fatal in critical condition. As of early March, 3300 health care workers were infected in China. In Italy, $20 \%$ of health care responders were infected. ${ }^{6}$ It directly affects the workforce required for smooth running of health institution. At individual level, it is cause of physiological and psychological stress. Awareness in terms of knowledge, attitude and practices of medical illnesses is essential for a doctor. Same holds true for pandemic of COVID-19. Adequate knowledge, appropriate attitudes and standard practices help in streamlining the management along with personal protection. Some studies have been conducted to evaluate data regarding knowledge, attitude and practices among various sections of society. One such study was conducted by Zhong et al in which participants were residents of Hubei province, China. The study summarized that gender and socioeconomic status affected the responses ${ }^{7}$. Another study was carried out on Iranian population regarding novel Corona virus outbreak ${ }^{8}$. Saqlain et al conducted KAP study among health care professionals including physicians, nurses and pharmacists. They observed positive attitude and sound practices. The results showed that age, experience and nature of job were significantly associated with the parameters of knowledge and practices'.

Considering the reported high infectivity of doctors, it is hypothesized that the knowledge, practice and attitude may be deficient. For this purpose, a questionnaire was designed to assess knowledge, attitude and practices of doctors regarding COVID-19. The target population was post graduate trainees and house officers who form vital and crucial part of health care delivery system. They are the force working at fore front in the pandemic due to novel Corona virus. The study was planned and carried out during initial period of pandemic. The rationale of the study was to identify and define deficiencies in knowledge, attitude and practices of post graduate trainees and house officers towards COVID-19.

\section{Data Collection Procedure}

This cross sectional study with web based survey was carried out among house officers and post graduate residents in tertiary care hospitals of Lahore, Pakistan from June 2020 to August 2020. After approval of Ethical Review Committee Allama Iqbal Medical College Lahore, data was collected via online questionnaire. Questionnaire was prepared using online Google Forms as a tool. Link was shared with participants through email, WhatsApp and Facebook. Clarity of language was assessed by running pilot study on 20 doctors. Informed consent was taken as part of questionnaire. Questionnaire was divided into sections. Demographic profile including age, gender, academic status, training duration and name of institution were documented. Questions were divided into sections pertaining to domains of knowledge, attitude and practice regarding COVID-19. Section on knowledge contained fourteen questions (K1-K14). Third section had three questions (A1-A3) related to attitude and fourth section had six questions concerned with practices (P1-P6). K1-K14 questions have choose the best option format. A1-A3 and P1-P6 have agree/disagree and yes/no format respectively.

\section{Data Analysis}

Data analysis was done with SPSS 24®(Armonk NY:IBM corp). Numerical variables like age was presented as mean \pm SD. For questions K1-K14, score of 1 was assigned to correct answer. Nominal variables like gender, academic status and responses for knowledge, attitude and practices were presented as frequency and percentages. Score of more than $50 \%$ was representative of positive attitude. Scores of three domains were correlated with gender, academic status and training duration by applying Pearson Correlation statistics. P value of $<0.05$ was considered significant.

\section{Results}

115 doctors responded to online survey. Mean age was 26.92 \pm 2.92 , (Fig-1) 62(53.9\%) were female and $53(46.1 \%)$ were male. Regarding the status of participants, $46(40 \%)$ were house officers and $69(60 \%)$ were post graduate residents. Inter-rater reliability was $85.6 \%$. There were fourteen questions 
related to cognitive assessment of covid infection. Maximum score was 12 and minimum was 4 . Mean score was 8.75 . Highest score of $12(85.7 \%)$ was noted in five $(4.3 \%)$ responders. Maximum correct response was for the most common clinical feature (99.1\%). Least response was for sequence of donning (Table-1). Considering the attitude, response to three questions was required. Participants had to choose between option "agree" and "disagree". It was observed that $82(71.3 \%)$ participants agreed that COVID-19 will be successfully controlled. 114(99.1\%) of participants were counselling patients and their attendants regarding preventive measures. Regarding query about whether personal information of positive cases should be made public, 64(55.7\%) agreed that it should be made public. In section regarding practice, six questions were asked and response was generated in form of options of "yes" or "no". Survey showed
Table 1: Correlation between Professional Status and the Domains

\begin{tabular}{llcccc} 
& & status & $\begin{array}{c}\text { Know- } \\
\text { ledge }\end{array}$ & $\begin{array}{c}\text { Prac } \\
\text {-tice }\end{array}$ & $\begin{array}{c}\text { Totaka } \\
\text {-ttitude }\end{array}$ \\
\hline status & Pearson & 1 & $-.326^{* *}$ & .055 & .081 \\
& Correlation & & & & \\
& Sig. (2-tailed) & & .000 & .559 & .392 \\
& N & 115 & 115 & 115 & 115 \\
Know- & Pearson & $-.326^{* *}$ & 1 & -.053 & -.011 \\
ledge & Correlation & & & & \\
& Sig. (2-tailed) & .000 & & .575 & .904 \\
& N & 115 & 115 & 115 & 115 \\
Prac-tice & Pearson & .055 & -.053 & 1 & .073 \\
& Correlation & & & & \\
& Sig. (2-tailed) & .559 & .575 & & .439 \\
& N & 115 & 115 & 115 & 115 \\
Totaka- & Pearson & .081 & -.011 & .073 & 1 \\
ttitude & Correlation & & & & \\
& Sig. (2-tailed) & .392 & .904 & .439 & \\
& N & 115 & 115 & 115 & 115 \\
**. Correlation is significant at the $\mathbf{0 . 0 1}$ level (2-tailed). \\
\hline
\end{tabular}

115 responses

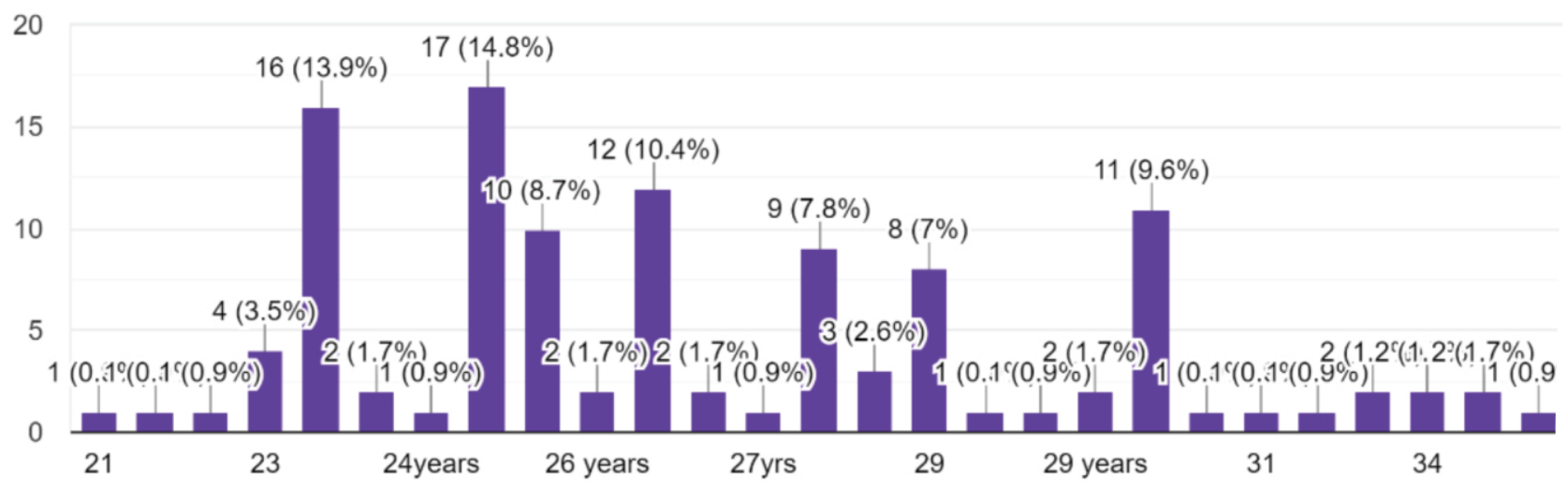

Figure-1 Age distribution among participants

that 59(51.3\%) answered in affirmative regarding recent visits to crowded places. $110(95.7 \%)$ wore mask when leaving home while $19(16.5 \%)$ had undergone training for nasopharyngeal sample collection. Only 45(39.1\%) had undergone training for doffing and donning. Contact with covid positive cases was documented in $37(32.2 \%)$ during data collection period. Scrubs were worn by $72(62.6 \%)$.

Pearson correlation was applied between the three domains and status of doctors. It was observed that postgraduate residents were significantly better in Covid-19 related knowledge $(\mathrm{p}<.05)$. Among the three domains, no significant relationship was observed.

\section{Discussion}

This study aimed at knowledge, attitude and practices of doctors regarding covid-19 outbreak Study was carried out at the time when Pakistan was experiencing first wave of COVID infection. Covid-19 pandemic has highlighted the importance of knowledge of emerging advances in medical field. Study was carried out at the time when Pakistan was experiencing first wave of COVID infection. Doctors are the front line workers. They have to manage the patients suffering from the disease. They themselves are vulnerable to fall prey to this viral infection. This highlights the importance of personal safety. Various countries have conducted such surveys. One study was carried out in Nepal. The study involved doctors, nurses and paramedics. Our 
study focused on doctors. $76 \%$ of participants had adequate knowledge, while in our study $4.3 \%$ scored $85.7 \%$. In Nepalese study, $54.7 \%$ and $78.9 \%$ had positive attitude and app-ropriate practice approach regarding COVID-19. ${ }^{10}$ Our study documented positive attitude though practice approach was not adequate. This fact can be explained by lack of awareness and training techniques. In another study conducted in a tertiary care hospital in Nepal, it was concluded that knowledge attitude and practice regarding COVID had positive relationship with age. ${ }^{11}$ In our study post graduate residents had significantly better knowledge than house officers.

A local perspective was expressed in a study carried out in Karachi. Study had 414 participants. Analysis showed sound knowledge (93.2\%), attitude and practice (88.7\%); though gaps were identified in certain fields ${ }^{12}$. Our study found that most of the participants lacked knowledge regarding social distancing or treatment options. Positive attitude was observed though practice domain reflected deficiency in training regarding preparation to deal with pandemic.

A study by Salman et al was carried out among health care professionals in which doctors were found to have better knowledge with overall score of $75.5 \%$. Our study involved two tiers of doctors i.e. house officers and post graduate residents and highest score was $85.7 \%$. In the same study practice skills were found to be satisfactory in $73.4 \%$ of participants. ${ }^{13}$

\section{Conclusion}

According to the response of the participants, positive attitude is present in most of the doctors though gaps have been identified in knowledge and practice that need improvement.

\section{Conflict of Interest: None}

\section{References}

1. Li Q, Guan X, Wu P, Wang X, Zhou L, Tong Y et al. Early Transmission Dynamics in Wuhan, China, of Novel Coronavirus-Infected Pneumonia. New England Journal of Medicine. 2020;382(13):1199-1207.

2. Voice from China. Chinese Medical Journal. 2020; 133(9):1012-1014.

3. Das P, Choudhuri T. Decoding the global outbreak of COVID-19: the nature is behind the scene. Virus Disease. 2020;. https://doi.org/10.1007/s13337-020-00605-y.

4. Coronavirus disease (COVID-19)-World Health Organization [Internet]. Who.int. 2021 [cited 23 1. Coronavirus disease (COVID-19) - World Health Organization [Internet]. Who.int. 2021 [cited 23 February 2021].
Available from: https://www.who.int/emergencies/ diseases/novel-coronavirus-2019? gclid $=$ CjwKC Ajwxev3BRBBEiwAiB_PWHKC8mLCiuPi1C5vL ew 1 KS evXk5pA-NNyA_Wn61sbQK3 mpIsb DIJOhoCjIUQAvD_2021].

5. [Internet]. Covid.gov.pk. 2021 [cited 1 July 2021]. Available from: http://covid.gov.pk/

6. Editorial. COVID-19: protecting health-care workers. Lancet. 2020; 395(10228):922. doi: 10.1016/ S01406736(20)30644-9.

7. Zhong B, Luo W, Li H, Zhang Q, Liu X, Li W et al. Knowledge, attitudes, and practices towards COVID19 among Chinese residents during the rapid rise period of the COVID-19 outbreak: a quick online crosssectional survey. International Journal of Biological Sciences. 2020;16(10):1745-1752.

8. Erfani A, Shahriarirad R, Ranjbar K, Mirahmadizadeh A, Moghadami M. Knowledge, Attitude and Practice toward the Novel Coronavirus (COVID-19) Outbreak: A Population-Based Survey in Iran. [Internet]. 2020 [cited 8 July 2020]; : Available from: https://www.who.int/ bulletin/online_first/20-256651.pdf.

9. Saqlain M, Munir M, Rehman S, Gulzar A, Naz S, Ahmed $\mathrm{Z}$ et al. Knowledge, Attitude and Practice among Healthcare Professionals regarding COVID19: A cross-sectional survey from Pakistan [Internet]. Medrxiv.org. 2020 [cited 8 July 2020]. Available from: https://www.medrxiv.org/content/10.1101/ 2020. 04.13.20063198v1.full.pdf

10. Tamang N, Rai P, Dhungana S, Sherchan B, Shah B, Pyakurel P et al. COVID-19: a National Survey on perceived level of knowledge, attitude and practice among frontline healthcare Workers in Nepal [Internet]. 2021 [cited 10 February 2021]. Available from: https:// bmcpublichealth.biomedcentral.com/articles/10.118 6/s12889-020-10025-8

11. Limbu D, Piryani R, Sunny A. Healthcare workers' knowledge, attitude and practices during the COVID19 pandemic response in a tertiary care hospital of Nepal. PLOS ONE. 2020;15(11):e0242126.

12. Saqlain M, Munir M, Rehman S, Gulzar A, Naz S, Ahmed $\mathrm{Z}$ et al. Knowledge, attitude, practice and perceived barriers among healthcare workers regarding COVID-19: a cross-sectional survey from Pakistan. Journal of Hospital Infection. 2020;105(3):419-423.

13. Salman M, Mustafa Z, Asif N, Zaidi H, Shehzadi N, Khan T et al. Knowledge, attitude and preventive practices related to COVID-19 among health professionals of Punjab province of Pakistan. The Journal of Infection in Developing Countries. 2020;14(07):707-712.

\section{Authors Contribution}

S.T: Conceptualization of Project, Writing of Manuscript, Literature Search

S.S: Statistical Analysis, Drafting, Revision, Data Collection 